\title{
SOLUTION OF NATURAL CONVECTION BOUNDARY LAYER Flow Above A Semi-Infinite Porous Horizontal Plate UNDER Similarity Transformations With SUCTION AND BLOWING
}

\author{
M. M. Touhid Hossain ${ }^{1,}$ Rita Mojumder ${ }^{2}$ and Mohammad Arif Hossain ${ }^{3}$ \\ Department of Mathematics \\ Khulna University of Engineering \& Technology, Khulna- 9203, Bangladesh \\ Email: mthosain@yahoo.co.in, rita_mojumder@yahoo.com, arifkhan63@hotmail.com
}

\begin{abstract}
In the present study we have confined our attention to the laminar boundary layer equations for the unsteady free convection flow over a heated horizontal semi-infinite porous plate by simplifying them using the Boussinesq approximation. Similarity requirements for an incompressible fluid are sought on the basis of detailed analysis in order to reduce the governing coupled partial differential equations into a set of ordinary differential equations. Numerical results are displayed graphically for some selected values of the controlling parameters provided by the similarity transformation. The influence of suction and blowing on the flow and temperature fields and other flow factors like skin friction and heat transfer coefficients are extensively investigated. It is found that a small value of suction or blowing play a vital role on the patterns of flow and temperature fields as well as on the coefficients of skin friction and heat transfer.
\end{abstract}

Keywords: Natural convection, Boussinesq approximation, Similarity transformation, Suction and blowing.

\section{Introduction}

The fluid flow caused only due to the density differences resulting from temperature gradients, without the assistance of any external force is termed as natural or free convection flow. Such a flow is generated due to the buoyancy effects which is observed in many heat transfer processes in nature and is applied in many technological applications. Furthermore, the solution of a system of coupled partial differential equations with boundary conditions is often difficult and even impossible with the usual classical method. Thus, it is imperative to reduce the number of variables from the system which reached in a stage of great extent. Similarity solution is one Date of submission : 05-01-2010 Date of acceptance :09-10-2010 of the important means for the reduction of a number of independent variables with simplifying assumptions and finally the system of partial differential equations reduces to a set of ordinary differential equations successfully. A vast literature of similarity solution has appeared in the area of fluid mechanics, heat transfer, and mass transfer, etc.

The theoretical, experimental and numerical analysis for the natural convection boundary layer flow about isothermal, vertical flat plates have been carried out widely by many authors (viz. [1, 2, 3, 4, 5, 6]). In 1978, Johnson and Cheng [7] examined the necessary and sufficient conditions under which similarity solutions exist for free convection boundary layers adjacent to flat plates in porous media. The solutions obtained in their work were more general than those appearing in the previous studies. Studies of the natural convection over a horizontal impermeable plate are available in $[8,9,10,11]$. It is also well established that the suction or injection of fluid through a horizontal surface can significantly modify the flow field and affect the heat transfer rate for forced as well as free convection flows. The study of natural convection on a horizontal plate with suction and blowing is of huge interest in many engineering applications, for instance, transpiration cooling, boundary layer control and other diffusional operations. Using the usual asymptotic approach, the similar solutions of the steady natural convection boundary layer for a non-similar flow situation on a horizontal plate with large suction approximation has been developed by Afzal and Hussain [12]. A detailed study on similarity solutions for free convection boundary layer flow over a permeable wall in a 
fluid saturated porous medium was carried out by Chaudhary et al. [13]. They have shown that the system depends on the power law exponent and the dimensionless surface mass transfer rate. They also examined the range of exponent under which the solution exists. With constant plate temperature and a particular distribution of blowing rate Clarke and Riley [14] obtained a special case of similarity solution, allowing variable fluid density. But there is still a shortage of accurate data for a wide range of both suction and blowing rate. Lin and $\mathrm{Yu}$ [15] presented a non-similar solution for the laminar free convection flow over a semi-infinite heated upward-facing horizontal porous plate with suitable transpiration rate as a power-law variation. Emphasis was given for an isothermal plate under the condition of uniform blowing or suction. Lately, using a parameter concerned pseudo-similarity technique of time and position coordinates, Cheng and Huang [16] studied the unsteady laminar boundary layer flow and heat transfer in the presence and absence of heat source or sink on a continuous moving and stretching isothermal surface with suction and blowing. In their analysis they paid attention on the temporal developments of the hydrodynamic and thermal characteristics after the sudden simultaneous changes in momentum and heat transfer. Recently, an analysis is performed by Aydin and Kaya [17] for the laminar boundary layer flow over a porous horizontal flat plate, particularly, to study the effect of uniform suction/injection on the heat transfer. Using the constant surface temperature as thermal boundary condition they also investigated the effect of Prandtl number on heat transfer.

In the present study we have confined our discussion about a complete similarity solution of the unsteady natural convection boundary layer flow above a heated horizontal semiinfinite porous plate and investigated the effects of suction and blowing on the flow and temperature fields and other important flow parameters like skin friction, heat transfer coefficients. Firstly, the governing differential equations relevant to the problem have been solved by using the similarity technique. The Boussinesq approximation is employed to deal with the possible requirements of unsteady solution. Similarity requirements for an incompressible fluid are sought on the basis of detailed analysis in order to reduce the governing coupled partial differential equations into a set of ordinary differential equations. Secondly, numerical solutions were displayed for some typical values of the established parameters. The effects of these parameters on several variables will also be exhibited in the analysis. We also have tried to predict the role of small suction or blowing velocity on these parameters concerned.

\section{Basic Equations}

The unsteady laminar two-dimensional boundary layer flows above a heated horizontal porous surface, maintained at a temperature different to that of the ambient fluid conditions are governed by the continuity, momentum and energy equations as follows:

$$
\begin{aligned}
& \frac{\partial \rho}{\partial t}+\frac{\partial}{\partial x}(\rho u)+\frac{\partial}{\partial y}(\rho v)=0 \\
& \frac{\partial u}{\partial t}+u \frac{\partial u}{\partial x}+v \frac{\partial u}{\partial y}=\frac{1}{\rho}\left(\rho-\rho_{0}\right) g_{x}-\frac{1}{\rho} \frac{\partial p}{\partial x} \\
& +\frac{1}{\rho} \frac{\partial}{\partial x}\left(\mu \frac{\partial u}{\partial x}\right)+\frac{1}{\rho} \frac{\partial}{\partial y}\left(\mu \frac{\partial u}{\partial y}\right) \\
& \frac{\partial v}{\partial t}+u \frac{\partial v}{\partial x}+v \frac{\partial v}{\partial y}=\frac{1}{\rho}\left(\rho-\rho_{0}\right) g_{y}-\frac{1}{\rho} \frac{\partial p}{\partial y} \\
& +\frac{1}{\rho} \frac{\partial}{\partial x}\left(\mu \frac{\partial v}{\partial x}\right)+\frac{1}{\rho} \frac{\partial}{\partial y}\left(\mu \frac{\partial v}{\partial y}\right) \\
& \rho c_{p} \frac{D T}{D t}=\frac{\partial}{\partial x}\left(k \frac{\partial T}{\partial x}\right)+\frac{\partial}{\partial y}\left(k \frac{\partial T}{\partial y}\right) \\
& +\left(u \frac{\partial p}{\partial x}+v \frac{\partial p}{\partial y}\right)+\mu\left[2\left\{\left(\frac{\partial u}{\partial x}\right)^{2}+\left(\frac{\partial v}{\partial y}\right)^{2}\right\}\right. \\
& \left.+\left(\frac{\partial u}{\partial y}+\frac{\partial v}{\partial x}\right)^{2}-\frac{2}{3}\left(\frac{\partial u}{\partial x}+\frac{\partial v}{\partial y}\right)^{2}\right]
\end{aligned}
$$

In order to derive the boundary layer equations, it is anticipated that the $v$ component of the velocity is small enough and assumed that the suction or blowing velocity normal to the surface has its order of magnitude $O\left(\operatorname{Re}^{-\frac{1}{2}}\right)$. Consequently, the flow outer the boundary layer is independent of $v_{w}$, so that, the boundary conditions regarding velocities at the surface is given by

$y=0 ; u=0, \quad v=v_{w}(x)$

The following non-dimensional variables are also introduced:

$$
\begin{aligned}
& t^{\prime}=\frac{U t}{L}, x^{\prime}=\frac{x}{L}, y^{\prime}=\mathrm{Re}^{\frac{1}{2}} \frac{y}{L}, u^{\prime}=\frac{u}{U}, \\
& v^{\prime}=\mathrm{Re}^{\frac{1}{2}} \frac{u}{U}, p^{\prime}=\frac{p}{\rho_{r} U^{2}}, \rho^{\prime}=\frac{\rho}{\rho_{r}}, \\
& \mu^{\prime}=\frac{\mu}{\mu_{r}}, k^{\prime}=\frac{k}{k_{r}}, c_{p}^{\prime}=\frac{c_{p}}{c_{p_{r}}}, T^{\prime}=\frac{T}{T_{r}}, \\
& g_{x}^{\prime}=\frac{g_{x}}{g}, g_{y}^{\prime}=\frac{g_{y}}{g}
\end{aligned}
$$




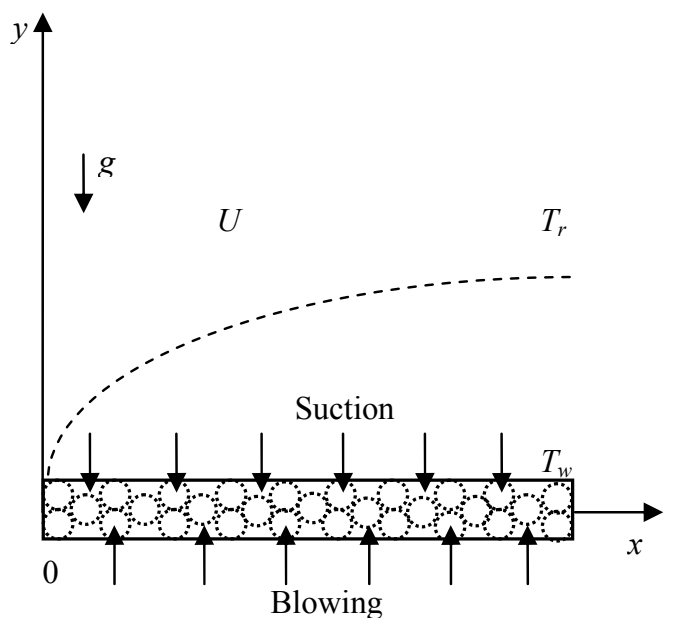

Fig. 1: Schematic Representation and Coordinate System of the Problem.

where dashed variables are non-dimensional and $U, L$ represent convenient characteristic velocity and length scales, $\operatorname{Re}=\frac{U L}{v_{r}}$ is a characteristic Reynolds number based on $U$ and $L$. Suffix $r$ refers to a convenient constant reference condition at a fixed point outside the boundary layer. The Cartesian co- ordinates $x$, $y$ are chosen to lie along and normal to the plate, $g_{x}$ and $g_{y}$ are the components of the gravity vector in the $x$ and $y$-directions. The perturbation pressure $\widetilde{p}$ is related to the absolute pressure $p$ by the equation $p=\tilde{p}+p_{0}, \quad$ where $\quad \frac{\partial p_{0}}{\partial x}=\rho_{0} g_{x}$, $\frac{\partial p_{0}}{\partial y}=\rho_{0} g_{y}$. Here suffix 0 is considered to denote conditions in a fluid at rest and $\widetilde{p}$ is termed as the motion pressure. The gradients of the hydrostatic pressure $p_{0}$ are balanced by the body force terms. Also, in the present investigation we are concerned with those boundary layer flows for which $\frac{\operatorname{Re}^{-\frac{1}{2}}}{\operatorname{Fr}}\left(1-\frac{\rho_{0}^{\prime}}{\rho^{\prime}}\right) g_{y}^{\prime} \square O(1)$, where $\operatorname{Fr}\left(=\frac{U^{2}}{g L}\right)$ is the characteristic Froude number. Since the variation in the buoyancy force normal to the surface is the only means of producing boundary layer motion, on a horizontal surface, the component of the buoyancy force parallel to the surface is zero, i.e., $g_{x}{ }^{\prime}=0$. In natural convection flow the order of magnitude of velocity created by the density differences across the boundary layer is determined as $U \square O\left\{\frac{\rho_{w}^{\prime}-\rho_{0}^{\prime}}{\rho_{w}^{\prime}} g_{y}^{\prime}\left(v_{r} L\right)^{\frac{1}{2}}\right\}^{\frac{2}{5}}$.

If $\mathrm{Re}$ is large then $\mathrm{Re}^{-1}$ is treated as very small in magnitude. The pressure gradient normal to the surface caused by the density difference generates the perturbation pressure field $\not p$ inside the boundary layer, $x^{\prime}$ variations of this field being sufficient to cause the motion in the boundary layer. For those cases in which $p_{0}$ is determined by the condition that a given function of state is constant, it can be shown that

$k d p_{0} \square O\left(\frac{l_{0} g \beta_{T}}{c_{p}}\right), d \rho_{0} \square O\left(\frac{l_{0} g \beta_{T}}{c_{p}}\right)$,

where $l_{0}$ represents the vertical scale of the flow field, which can considerably be taken to be the maximum boundary layer thickness and $k=\frac{1}{\rho}\left(\frac{\partial \rho}{\partial p}\right)_{T}, \beta_{T}=\frac{1}{\rho}\left(\frac{\partial \rho}{\partial T}\right)_{p}$.

Consequently, with the additional provision $k_{r} \rho_{r} U^{2}<<1$, it follows that $\rho=\rho(T)$, $\rho_{0}=\rho_{r}$, so that variations in $\rho_{0}$ due to hydrostatic relations can be ignored .

Since the present study is concerned solely with the possible self-similar flow situations for a Boussinesq fluid, without loss of generality we have been introduced the effect of buoyancy by means of the Boussinesq approximation. Thus, fluid property variations other than the essential density variation are ignored completely in this approximation. The density difference $\left(=\rho_{r}-\rho\right)$ is indispensable to the free convection motion and must be retained where they appear in the body force term (i.e., term multiplied by $g$, the acceleration due to gravity), but elsewhere the density variation is considered to be small enough and is to be neglected. In view of the above discussions and omitting the dashes and ignoring the pressure and viscous work contributions because of the relatively small 
Eckert number $\mathrm{E}\left(=\frac{U^{2}}{c_{p} \Delta T}\right) \quad$ usually

encountered in the free convection flow, the governing boundary layer equations of laminar two-dimensional unsteady flow over a semiinfinite heated horizontal porous surface in dimensional form are simplified to the following form:

$$
\begin{aligned}
& \frac{\partial u}{\partial x}+\frac{\partial v}{\partial y}=0 \\
& \frac{\partial u}{\partial t}+u \frac{\partial u}{\partial x}+v \frac{\partial u}{\partial y}+\frac{1}{\rho_{r}} \frac{\partial p}{\partial x}=v_{r} \frac{\partial^{2} u}{\partial y^{2}} \\
& \frac{\partial p}{\partial y}=\left(\rho-\rho_{r}\right) g \\
& \frac{\partial T}{\partial t}+u \frac{\partial T}{\partial x}+v \frac{\partial T}{\partial y}=\frac{v_{r}}{\operatorname{Pr}} \frac{\partial^{2} T}{\partial y^{2}}
\end{aligned}
$$

where $g_{y}= \pm g, v_{r}=\frac{\mu_{r}}{\rho_{r}}$ are all constants, $\operatorname{Pr}\left(=\frac{\mu_{r} c_{p_{r}}}{k_{r}}\right)$ is the characteristic Prandtl number of the flow and $\rho \propto \frac{1}{T}$. There are, however, boundary conditions, which is imposed in order to determine the solution of the boundary layer equations (7) - (10).

i) $u=0, v=v_{w}$ at $y=0$,

ii) $T=T_{w}$ at $y=0 \Rightarrow \theta=1$ at $y=0$

iii) $u=0$ when $y \rightarrow \infty$ and

iv) $T=T_{r} \Rightarrow \theta=0$ when $y \rightarrow \infty$

(suffix ' $w$ ' represents the condition at the surface of the plate and suffix ' $r$ ' is the constant reference condition in the fluid at rest exterior the boundary layer).

\section{Similarity Transformations}

In order to reduce the above system of equations into convenient forms, we adopt the method of seeking similarity solutions. Hence the following substitutions are introduced:

$$
\begin{aligned}
& \tau=t, \xi=x, \phi=\frac{y}{\gamma(x, t)}, u=\frac{\partial \psi}{\partial y}, v=-\frac{\partial \psi}{\partial x}, \\
& \left(T-T_{r}\right)=\Delta T(\tau, \xi) U(\tau, \xi) \theta(\tau, \xi, \phi), \\
& \Delta T=T_{w}(\tau, \xi)-T_{r}, \\
& \psi(\tau, \xi, \phi)=\gamma(\tau, \xi) U(\tau, \xi) F(\tau, \xi, \phi), \\
& \tilde{p}=p(\tau, \xi) G(\tau, \xi, \phi), \\
& \left(\rho-\rho_{r}\right)=-\rho_{r} \beta_{T} \Delta T(\tau, \xi) \theta(\tau, \xi, \phi)
\end{aligned}
$$

Guided by the idea of the similarity procedure, we also use the traditional substitution: $\int_{0}^{\phi} \frac{u}{U(x, t)} d \phi_{1}=F(\tau, \xi, \phi)$

Then we obtain:

$\psi(\tau, \xi, \phi)=\gamma U F(\tau, \xi, \phi)+\psi(\tau, \xi, 0)$, $u=U F_{\phi}$ and $v=\gamma_{\xi} U \phi F_{\phi}-(\gamma U F)_{\xi}+v_{w}$

Here $v_{w}=-\frac{\partial \psi(\tau, \xi, 0)}{\partial \xi}$ represents the nonzero wall velocity called the suction or blowing velocity normal to the porous surface, so that fluid can either be sucked or blown through it. Physically, $v_{w}<0$ and $v_{w}>0$ represent the suction and blowing velocity through the porous surface, respectively. For uniform suction (or blowing) $v_{w}=$ constant . However, $v_{w}=0$ implies that the surface is impermeable to the fluid. In view of above transformations, equations (7) to (10) become:

$v_{r} F_{\phi \phi \phi}+a_{0} \phi F_{\phi \phi}+\frac{1}{2}\left(a_{1}+a_{2}\right) F F_{\phi \phi}-a_{2} F_{\phi}^{2}$

$-a_{4} F_{\phi}+a_{9} F_{\phi \phi}=a_{5} G-a_{6} \phi G_{\phi}$

$G_{\phi}=\bar{a}_{6} \theta$

$\frac{v_{r}}{\operatorname{Pr}} \theta_{\phi \phi}+a_{0} \phi \theta_{\phi}+\frac{1}{2}\left(a_{1}+a_{2}\right) F \theta_{\phi}-\left[\left(a_{4}+\right.\right.$

$\left.\left.a_{7}\right)+\left(a_{2}+a_{8}\right) F_{\phi}\right] \theta+a_{9} \theta_{\phi}=0$

where $F(\tau, \xi, \phi), \theta(\tau, \xi, \phi)$ and $G(\tau, \xi, \phi)$

are assumed at this stage to be functions of $\phi$ alone with:

i) $\left.\left.a_{0}=\gamma \gamma_{\tau}, i i\right) a_{1}=\left(\gamma^{2} U\right)_{\xi}, i i i\right) a_{2}=\gamma^{2} U_{\xi}$,

iv) $a_{3}=\gamma(\gamma U)_{\xi}=\frac{1}{2}\left(a_{1}+a_{2}\right)$,

v) $\left.a_{4}=\frac{\gamma^{2} U_{\tau}}{U}, v i\right) a_{5}=\frac{\gamma^{2}}{\rho_{r} U} \frac{\partial p}{\partial \xi}$,

vii) $a_{6}=\frac{\gamma \gamma_{\xi} p}{\rho_{r} U}$, viii $) \bar{a}_{6}=-\frac{\gamma \rho_{r} \beta_{T} g \Delta T}{p(\tau, \xi)}$,

ix) $\left.a_{7}=\gamma^{2}(\log \Delta T)_{\tau}, x\right) a_{8}=\gamma^{2} U(\log \Delta T)_{\xi}$

and $x i$ ) $a_{9}=-\gamma v_{w}$

The transformed boundary conditions are now:

$$
\begin{gathered}
F(0)=F_{\phi}(0)=0, F_{\phi}(\infty)=0 ; \\
\theta(0)=1, \theta(\infty)=0
\end{gathered}
$$


The equations (17) furnish us with the conditions under which similarity solutions are obtained provided that all a's must be constants and thus equations (14) to (16) will finally become non-linear ordinary differential equations in the limiting situations for the remaining variable other than the similarity variable. Consequently, the relations given by equations (17) are the treated conditions which provide us the equations for $U(\tau, \xi)$ and $\gamma(\tau, \xi)$, the scale factors for the velocity component and the ordinate. Uniquely, these scale factors together with the suction or blowing parameter will determine the flow characteristics of the boundary layer. We shall now proceed to find $U, \gamma$ and consequently the suction velocity $v_{w}$ for the possible requirements of similarity solution in the case of Boussinesq fluid. In view of conditions $(i)-$ (v) in equation (17), we have $\gamma^{2} U=a_{1} \xi+A(\tau)$ and $\gamma^{2}=2 a_{0} \tau+B(\xi)$, where $A(\tau)$ is either a function of $\tau$ or constant and $B(\xi)$ is either a function of $\xi$ or constant. The above two relations yield $\frac{d A}{d \tau} \cdot \frac{d B}{d \xi}=\left(2 a_{0}+a_{4}\right)\left(a_{1}-a_{2}\right)$

Therefore, the forms of the similarity equations, the scale factors $U$ and $\gamma$ entirely depend on the equation (19).

\subsection{Similarity Cases}

Equation (19) yields possibilities of four similarity cases noted below and for the sake of brevity we have discussed the first case only.

(A) both $\frac{d A}{d \tau}$ and $\frac{d B}{d \xi}$ are finite constants,

(B) both $\frac{d A}{d \tau}$ and $\frac{d B}{d \xi}$ are zero,

(C) $\frac{d A}{d \tau} \neq 0$, but $\frac{d B}{d \xi}=0$ and

(D) $\frac{d A}{d \tau}=0$, but $\frac{d B}{d \xi} \neq 0$.

Case A: When both $\frac{d A(\tau)}{d \tau}$ and $\frac{d B(\xi)}{d \xi}$ are finite consents, then we have $U=U_{0}$ (constant) and $\gamma^{2}=2 a_{0} \tau+\frac{a_{1}}{U_{0}} \xi+\frac{C}{U_{0}}$, where $C$ is a constant. Substituting these in the conditions $(i)-(x)$ of equation (17) yields the relations between the constants as follows:

$a_{0}, a_{1}$ are arbitrary and $a_{2}=a_{4}=a_{5}=0$

$a_{3}=\frac{a_{1}}{2}, a_{7}=2 a_{0}, a_{8}=a_{1}, a_{6}$ and $\bar{a}_{6}$ are disposable constants. Substituting the constants and choosing $F=\alpha_{1} f, \phi=\alpha_{2} \eta \quad$ and $G=\alpha_{3} \bar{g}$ the above equations (14) to (16) reduce to:

$$
\begin{gathered}
f_{\eta \eta \eta}+\left(\eta+\beta f+f_{w}\right) f_{\eta \eta}+\eta \bar{g}_{\eta}+=0 \\
\bar{g}_{\eta}=\left(\frac{U_{F}}{U_{0}}\right)^{\frac{5}{2}} \theta \\
\operatorname{Pr}^{-1} \theta_{\eta \eta}+\left(\eta+\beta f+f_{w}\right) \theta_{\eta} \\
-2\left(1+\beta f_{\eta}\right) \theta=0
\end{gathered}
$$

where it is also chosen that

$$
\begin{aligned}
& \alpha_{1}=\alpha_{2}, \frac{a_{0} \alpha_{1}^{2}}{v_{r}}=1, \frac{a_{6} \alpha_{1}^{2} \alpha_{3}}{v_{r}}=1, \frac{a_{1}}{2 a_{0}}=\beta, \\
& -\frac{\gamma v_{w}}{\sqrt{a_{0} v_{r}}}=f_{w} \text { and } \\
& U_{F}=\left[\sqrt{2} \beta\left(-g \beta_{T} \Delta T\right)\left(v_{r} \times L\right)^{\frac{1}{2}}\right]^{\frac{2}{5}}, \text { which is }
\end{aligned}
$$
usually defined as the free convection velocity associated with the local characteristic length:

$$
L=U_{0}\left(\tau+\tau_{0}\right)+\beta\left(\xi+\xi_{0}\right) .
$$

Since we are concerned with a purely free convection flow, without loss of generality we may put $U_{F}=U_{0}$ and hence the similarity equations are:

$$
\begin{aligned}
& f_{\eta \eta \eta}+\left(\eta+\beta f+f_{w}\right) f_{\eta \eta}+\eta \bar{g}_{\eta}=0 \\
& \bar{g}_{\eta}=\theta \\
& \operatorname{Pr}^{-1} \theta_{\eta \eta}+\left(\eta+\beta f+f_{w}\right) \theta_{\eta} \\
& \quad-2\left(1+\beta f_{\eta}\right) \theta=0
\end{aligned}
$$

ubject to the transformed boundary conditions:

$$
\begin{aligned}
& f(0)=f_{\eta}(0)=f_{\eta}(\infty)=0 ; \\
& \theta(0)=1, \theta(\infty)=0 ;
\end{aligned}
$$

$\bar{g}_{n}(0)=1, \bar{g}_{n}(\infty)=0$

where the boundary conditions for $\bar{g}$ is obtained form that described for $\theta$ according to equation (24). The velocity components, the skin friction and the local heat transfer 
coefficients associated with the equations (23) - (25) are given by

$$
u=U_{F} f_{\eta}(\eta)
$$$$
-v=\frac{v_{r} \beta R_{F}^{\frac{1}{2}}}{\sqrt{2} L}\left[f(\eta)-\eta f_{\eta}(\eta)\right]-v_{w},
$$

$\tau_{w}=\frac{\mu U_{F} R_{F}^{\frac{1}{2}}}{\sqrt{2} L} f_{\eta \eta}(0)$

and

$q_{w}=-\frac{k \Delta T R_{F}^{\frac{1}{2}}}{\sqrt{2} L} \theta_{\eta}(0)$.

\section{Numerical Solution and Discussion}

To obtain the solution of the (23) - (25) having boundary conditions (26), we have used the shooting method. It is clear that the number of initial conditions are not sufficient to obtain the particular solution of the differential equations, so we require to assume additional missing/unspecified initial conditions. These are to be so assumed that the solution of the outer prescribed points also matches. With all the initial conditions (given and assumed) the equations are integrated in steps. If match is not found at the outer end then another set of initial conditions are considered. This trial and error process is taken care through Nachtsheim-Swigert iteration technique. The solution thus obtained in terms of the similarity variables are plotted and tabulated. The effect of suction parameter $f_{w}, \beta$ and Prandtl number Pr on velocity $f_{\eta}$, temperature $\theta$ and pressure $\bar{g}$ are plotted in Fig. 2 through Fig. 10. Also their effects on the coefficient of skin-friction and heat transfer coefficient are tabulated in Table- 1 and Table- 2, respectively. To observe the effect of $f_{w}, \beta$ and $\operatorname{Pr}$ are kept as constants (0.33 and 0.71 respectively). Similarly, to observe the effect of $\beta$ and $\operatorname{Pr}, f_{w}$ and $\operatorname{Pr}$ and $f_{w}$ and $\beta$ respectively are kept as constants. In all cases where $f_{w}$ is kept as constant, its value is chosen as 0.3, similarly, when $\operatorname{Pr}$ is kept constant, its value is chosen as 0.71 . But to observe the effect of $f_{w}, \beta$ is chosen as 0.33 , whereas, it is chosen as -0.5 to observe the effect of Pr.

Fig. 2 to Fig. 4 represent the effect of $f_{w}, \beta$ and $\operatorname{Pr}$ on the velocity profiles respectively. From figures it is observed that the velocity decreases with the increase in the controlling parameters, i.e., the velocity decreases with the increase in $f_{w}$ (for fixed $\beta$ and $\operatorname{Pr}$ ) or increase in $\beta$ (for fixed $f_{w}$ and $\operatorname{Pr}$ ) etc.

Fig. 5 to Fig. 7 represent the effect of $f_{w}, \beta$ and $\operatorname{Pr}$ on the temperature profiles respectively. The effect of the controlling parameters on temperature is the same as that on the velocity, i.e., with their increase (increasing one of them keeping the other two as fixed), $\theta$ decreases.

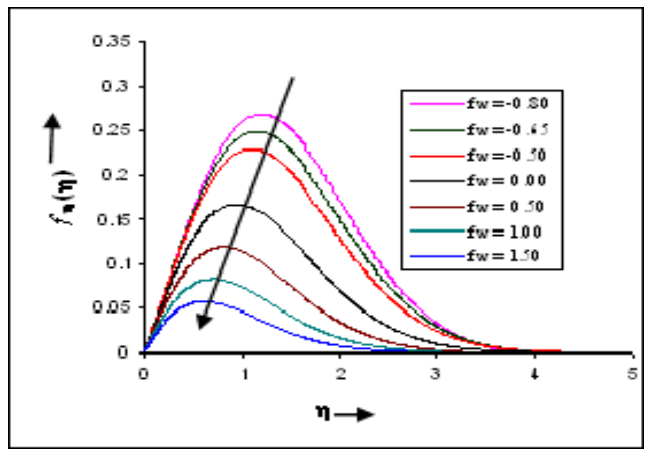

Fig. 2: Velocity profiles for different values of the suction parameter $f_{w}$ with fixed values of $\beta$ and $\operatorname{Pr}$

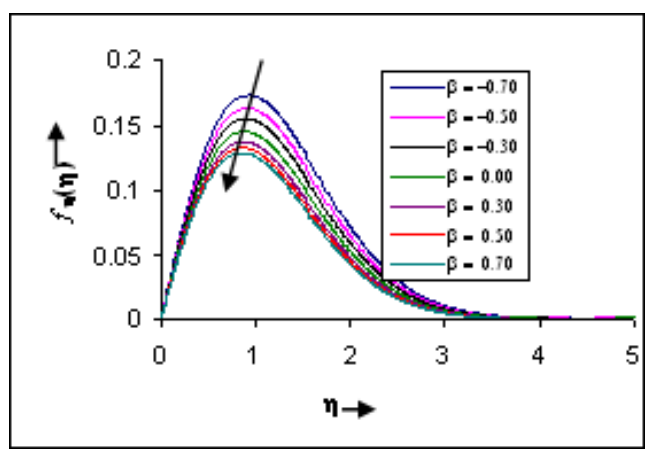

Fig. 3: Velocity profiles for different values of the parameter $\beta$ with fixed values of $f_{w}$ and $\operatorname{Pr}$

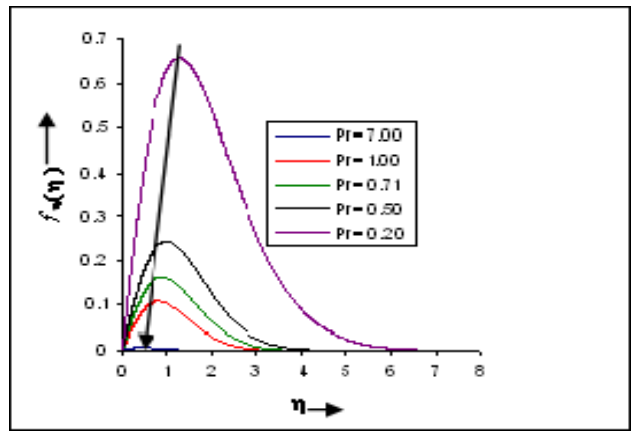

Fig. 4: Velocity profiles for different values of the Prandtl number $\operatorname{Pr}$ with fixed values of $f_{w}$ and $\beta$ 


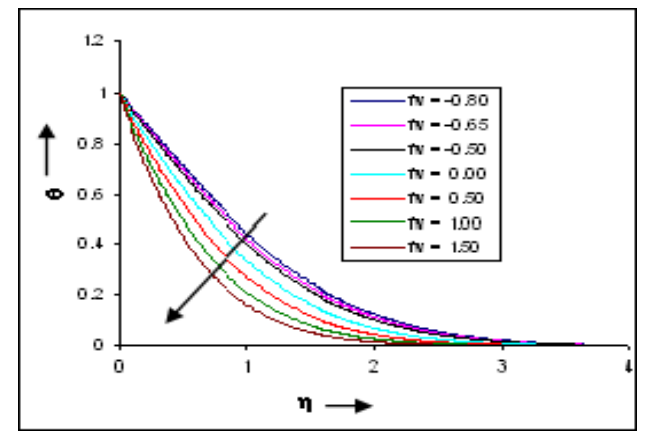

Fig. 5: Temperature profiles for different values of the suction parameter $f_{w}$ with fixed values of $\beta$ and $\operatorname{Pr}$

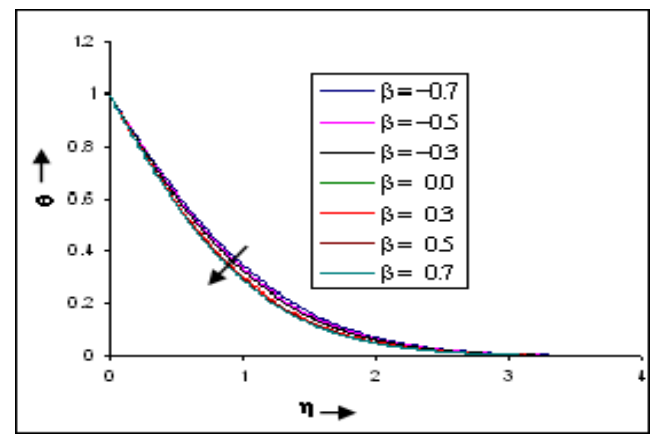

Fig. 6: Temperature profiles for different values of the parameter $\beta$ with fixed values of $f_{w}$ and $\operatorname{Pr}$

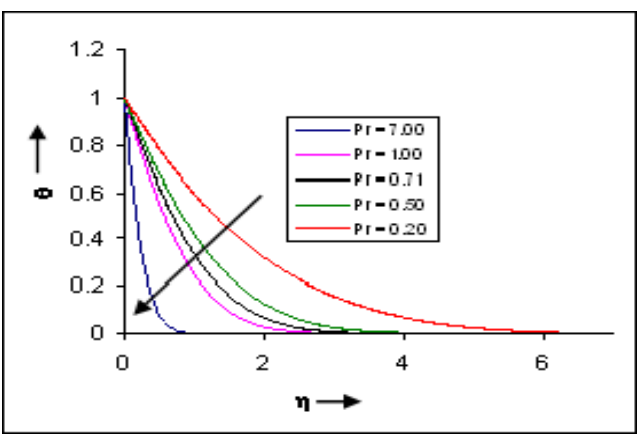

Fig. 7: Temperature profiles for different values of the Prandtl number Pr with fixed values of $f_{w}$ and $\beta$

Fig. 8 to Fig. 10 representing the effect of $f_{w}, \beta$ and $\operatorname{Pr}$ respectively, but on the pressure variable $\bar{g}$. In these cases their effects are reversed i.e., with the increase in the controlling parameters, pressure increases. This increase-increase behaviour is observed for all three parameters.

Fig. 9: Pressure distributions for different values of the Prandtl number Pr with fixed values of $f_{w}$ and $\beta$

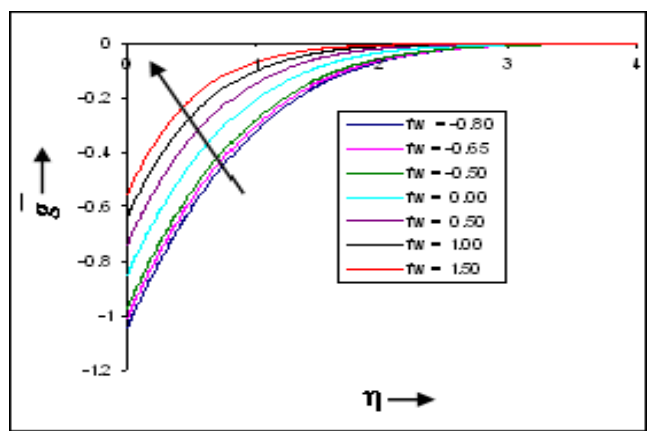

Fig. 8: Pressure distributions for different values of the suction parameter $f_{w}$ with fixed values of $\beta$ and Pr

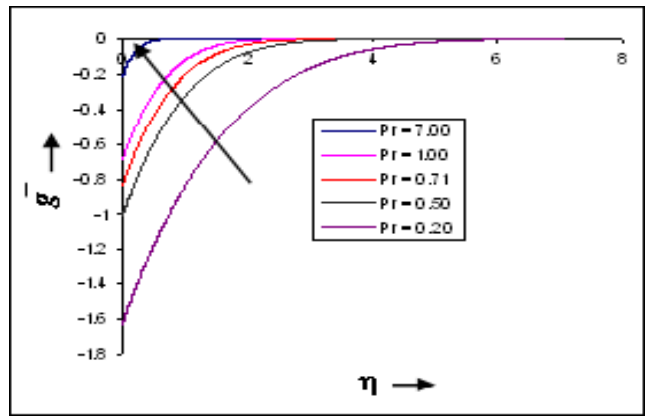

Table 1: Variation of the coefficient of skin friction with $f_{w}, \beta$ and pr

\begin{tabular}{|c|l|l|l|l|l|}
\hline \multicolumn{2}{|c|}{$\begin{array}{c}\text { Values Proportional to the coefficient of } \\
\text { skin-friction with the variation of }\end{array}$} \\
\hline $\begin{array}{c}f_{\mathrm{w}} \\
\text { (for } \beta=0.33 \\
\text { and } \\
\operatorname{Pr}=0.71)\end{array}$ & \multicolumn{2}{|c|}{$\begin{array}{c}\beta \\
\text { (for } f_{w}=0.3 \\
\text { and } \\
\operatorname{Pr}=0.71)\end{array}$} & \multicolumn{2}{|c|}{$\begin{array}{c}\text { Pr } \\
\text { (for } \beta=0.33 \\
\text { and } \\
\left.f_{w}=0.3\right)\end{array}$} \\
\hline$f_{\mathrm{w}}$ & $f_{\eta \eta}(0)$ & $\beta$ & $f_{\eta \eta}(0)$ & $\operatorname{Pr}$ & $f_{\eta \eta}(0)$ \\
\hline-0.80 & 0.3131 & -0.70 & 0.3413 & 0.20 & 0.9698 \\
\hline-0.65 & 0.3110 & -0.50 & 0.3265 & 0.50 & 0.4442 \\
\hline-0.50 & 0.3060 & -0.30 & 0.3143 & 0.71 & 0.3265 \\
\hline 0.00 & 0.3019 & 0.00 & 0.2992 & 1.00 & 0.2399 \\
\hline 0.50 & 0.2723 & 0.30 & 0.2873 & 7.00 & 0.0320 \\
\hline 1.00 & 0.2355 & 0.50 & 0.2800 & - & - \\
\hline 1.50 & 0.1977 & 0.70 & 0.2739 & - & - \\
\hline
\end{tabular}




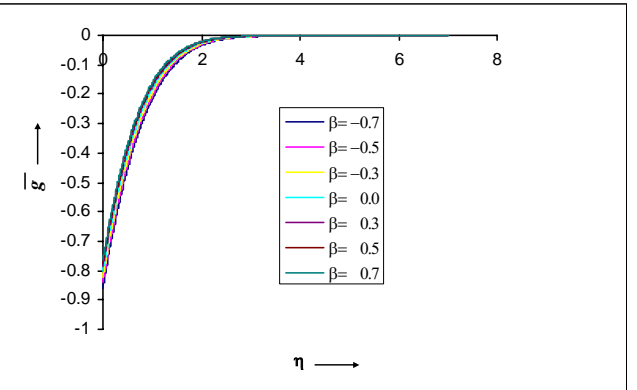

Fig. 10: Pressure distributions for different values of the parameter $\beta$ with fixed values of $f_{w}$ and $\operatorname{Pr}$

An important point is to be noted here that for the chosen fixed values of $\beta$ and $\operatorname{Pr}$ when $f_{w}$ is assigned any values less than -0.81 , the behaviour of the flow become chaotic i.e., turbulence is observed for $f_{w}<-0.81$. All the three variables we have tried to observe show the same behaviour.

The values proportional to the coefficient of skin-friction are tabulated in Table- 1. From this table the effect of $f_{w}, \beta$ and $\operatorname{Pr}$ on the skinfriction can be observed. It is seen that for fixed $\beta$ and $\operatorname{Pr}$ with the increase in the $f_{w}$, the coefficient of skin-friction decreases. The same behaviour is being observed for the other controlling parameters (increasing one of them keeping the other two as fixed).

Table 2 contains the values proportional to the heat transfer coefficient. The effect of the controlling parameters on it is as that to the skin-friction i.e., with the increase in the controlling parameters the coefficient of heat transfer decreases.

\section{Conclusion}

With the use of the similarity variables, the governing equations of the unsteady natural convection boundary layer flow is reduced to a set of ordinary differential equations. Four different similarity cases arise with the choice of $\frac{d A}{d \tau}$ and $\frac{d B}{d \xi}$ either zero or constant. Similarity solution for one case is being studied in this paper. It is being observed that the similarity variables related to the velocity and temperature have inverse relations with the controlling parameters. Whereas the variables related to the pressure has direct relation with the controlling parameters. It is also observed that the coefficient of skin-friction and heat transfer have also inverse relationship with the controlling parameters. It may be noted here that the flow became turbulent for $f_{w}<-0.81$. Further study is necessary to solve the rest cases.

Table 2: Variation of the heat transfer coefficient with $f_{w}, \beta$ and pr

Values Proportional to the heat transfer coefficient with the variation of

\begin{tabular}{|c|c|c|c|c|c|}
\hline \multicolumn{2}{|c|}{$\begin{array}{c}f_{\mathrm{w}} \\
\text { (for } \beta=0.33 \\
\text { and } \\
\operatorname{Pr}=0.71 \text { ) }\end{array}$} & \multicolumn{2}{|c|}{$\begin{array}{c}\beta \\
\text { (for } f_{w}=0.3 \\
\text { and } \\
\operatorname{Pr}=0.71 \text { ) }\end{array}$} & \multicolumn{2}{|c|}{$\begin{array}{c}\operatorname{Pr} \\
\text { (for } \beta=0.33 \\
\text { and } \\
f_{w}=0.3 \text { ) }\end{array}$} \\
\hline$f_{\mathrm{w}}$ & $\theta_{\eta}(0)$ & $\beta$ & $\theta_{\eta}(0)$ & $\operatorname{Pr}$ & $\theta_{\eta}(0)$ \\
\hline-0.80 & -0.5332 & -0.70 & -0.8165 & 0.20 & -0.4560 \\
\hline-0.65 & -0.5765 & -0.50 & -0.8396 & 0.50 & -0.6963 \\
\hline-0.50 & -0.6218 & -0.30 & -0.8597 & 0.71 & -0.8396 \\
\hline 0.00 & -0.7922 & 0.00 & -0.8860 & 1.00 & -1.0181 \\
\hline 0.50 & -0.9967 & 0.30 & -0.9087 & 7.00 & -3.0320 \\
\hline 1.00 & -1.2353 & 0.50 & -0.9223 & - & - \\
\hline 1.50 & -1.5021 & 0.70 & -0.9352 & - & - \\
\hline
\end{tabular}

\section{References}

[1] E. Schmidt and W. Bechmann, "Das Temperature-und Geschwidigkeitsfeld vor einer Wärme abgebenden senkrechter platte bei natürlicher convection", Tech. Mech. u. Termodynamik, Bd, 1(10), 1930, pp. 341-349.

[2] E. R. G. Eckert and E. E. Soehngen, "Studies on heat transfer in laminar free convection with Zehnden-Mech interferometer", Tech. Rept. 5747, ATI 44580, Air Materiel Command, Dayton, Ohio, 1948.

[3] S, Ostrach, "New aspects of natural convection heat transfer", Trans. ASME, Vol. 75, 1953, pp. 1287.

[4] W. E. Stewart, "Asymptotic calculation of free convection in Laminar Three-dimensional systems", Int. J. Heat Mass Transfer, Vol. 14, 1971, pp. 1013-1031.

[5] Suwono, A., Laminar free convection Boundary Layer in three-dimensional systems, Int. J. Heat Mass Transfer, Vol. 23, 1980, pp. 53-61.

[6] J. H. Merkin, "A note on the similarity solutions for free convection on a vertical plate", Journal of Engineering Mathematics, Vol. 19, 1985, pp. 189-201.

[7] C. H. Johnson and P. Cheng, "Possible similarity solutions for free convection boundary layers adjacent 
to flat plates in porous media", Int. J. Heat Mass Transfer, Vol. 21, 1978, pp. 709-718.

[8] K. Stewartson, "On the free convection from a horizontal plate", Zeitschrift für Angewandte Mathematik und Physik (ZAMP), Vol. 9a, 1958, pp. 276-282.

[9] W. N. Gill, D. W. Zeh and E. Del-Casal, "Free convection on a horizontal plate", Zeitschrift fur Angewandte Mathematik und Physik (ZAMP), Vol. 16, 1965, pp. 539-541.

[10] Z. Rotem and L. Claassen, "Natural convection above unconfined horizontal surfaces", Journal of Fluid Mechanics, Vol. 39, 1969a, pp. 173-192.

[11] L. Pera and B. Gebhart, "Natural convection boundarylayer over horizontal and slightly inclined surfaces", International Journal of Heat and Mass Transfer, Vol. 16, 1973, pp. 1131-1146.

[12] N. Afzal and T. Hussain, "Effects of large suction in natural convection boundary layer on a horizontal plate", Wärme-und Stoffübertragung, Vol. 20, 1986, pp. 203-206.
[13] M. A. Chaudhary, J. H. Merkin and I. Pop, "Similarity solutions in free convection boundary layer flows adjacent to vertical permeable surfaces in porous media", Eur. J. Mech., B/Fluids, Vol. 14(2), 1995, pp. 217-237.

[14] J. S. Clarke and N. Riley, "Natural convection induced in a gas by the presence of a hot porous horizontal surface", Quarterly Journal of Mechanics and Applied Mathematics, Vol. 28(4), 1975, pp. 373-396.

[15] Lin, H. T., Yu, W. S., Free convection on a horizontal plate with blowing and suction, Journal of Heat Transfer, (1988), 110, pp. 793796.

[16] W. T. Cheng and C. N. Huang, "Unsteady flow and heat transfer on an accelerating surface with blowing or suction in the absence and presence of a heat source or sink", Chemical Engineering Science, Vol. 59, 2004, pp. 771-780.

[17] O. Aydin and A. Kaya, "Laminar boundary layer flow over a horizontal permeable flat plate", Applied Mathematics and Computation, Vol. 161, 2005, pp. 229-240. 